Research Article

\title{
Iron Homeostasis in Patients with Nonalcoholic Fatty Liver Disease
}

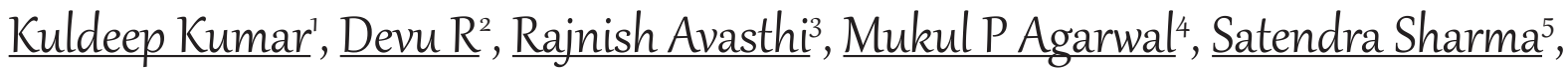 \\ Anupama Tandon ${ }^{6}$
}

${ }^{1}$ Assistant Professor, ${ }^{2}$ Post Graduate Resident, ${ }^{3}$ Professor \& Head, ${ }^{4}$ Professor, Department of Medicine, UCMS \& GTB Hospital, Delhi, India.

${ }^{5}$ Director Professor, Department of Pathology, UCMS \& GTB Hospital, Delhi, India.

${ }^{6}$ Associate Professor, Department of Radiodiagnosis, UCMS \& GTB Hospital, Delhi, India.

DOI: https://doi.org/10.24321/2349.7181.202107

I $\quad \mathbf{N} \quad \mathbf{F} \quad \mathbf{O}$

\section{Corresponding Author:}

Kuldeep Kumar, Department of Medicine, UCMS \& GTB Hospital, Delhi, India.

E-mail Id:

drkuldeep74@gmail.com

Orcid Id:

https://orcid.org/0000-0001-6246-5573

How to cite this article:

Kumar K, Devu R, Avasthi R, Agarwal MP, Sharma $S$, Tandon A. Iron Homeostasis in Patients with Nonalcoholic Fatty Liver Disease. J Adv Res Med. 2021; 8(2): 6-10.

Date of Submission: 2021-06-02

Date of Acceptance: 2021-06-29

\section{$\begin{array}{llllllll}\mathbf{A} & \mathbf{B} & \mathbf{S} & \mathbf{T} & \mathbf{R} & \mathbf{A} & \mathbf{C} & \mathbf{T}\end{array}$}

Introduction: Serum ferritin correlates with hepatic iron deposition in patients with Non Alcoholic Fatty Liver Disease (NAFLD). Iron removal improves insulin sensitivity thereby delaying the onset of type 2 diabetes mellitus, improves liver function and NAFLD histology.

Objectives: This study was done to assess the iron status in NAFLD patients and its association with fibrosis and metabolic syndrome in NAFLD.

Material and Method: The study was conducted in the Department of Medicine, GTB Hospital, Delhi from November 2017 - October 2019. It was an Analytical, comparative study. Forty patients with NAFLD were selected in the study group and forty healthy subjects with no evidence of fatty liver on ultrasound were selected in the control group. The level of iron parameters recorded in NAFLD patients and the control group were compared using independent $t$ test and $p$ value of $<0.05$ was considered statistically significant. Assessment of association of various parameters of iron status with the presence of Metabolic Syndrome and fibrosis in NAFLD patients was done using the Chi-Square test.

Result: The parameters of iron status namely serum iron, serum ferritin, transferrin saturation and serum hepcidin were found to be higher in NAFLD patients when compared to controls. This study also observed that there was a statistically significant positive association of serum ferritin with the presence of MetS in NAFLD patients. The study also found that serum hepcidin and serum ferritin have a significant positive association with fibrosis in NAFLD.

Discussion: Assessment of iron status in NAFLD may help in early identification of individuals at high risk of fibrosis and other complications like Mets. Application of iron removal strategies like phlebotomy in such patients might improve IR and NAFLD.

Keywords: Serum Ferritin, Fibrosis, Phlebotomy Metabolic Syndrome (MetS), NAFLD 


\section{Introduction}

Non-Alcoholic Fatty Liver Disease (NAFLD) is the most common cause of liver disease today. It is also considered the hepatic manifestation of Metabolic Syndrome (MetS), with Insulin Resistance (IR) being the determining factor in its pathophysiology. ${ }^{1}$ The prevalence of NAFLD is rapidly increasing along with the growing epidemic of obesity and diabetes affecting more than one-quarter of adults in the world. $60 \%$ of diabetic patients and $90 \%$ of the obese people have NAFLD. ${ }^{2}$ In India, the prevalence of NAFLD ranges from $8.7 \%$ to $32.6 \%$ in rural and urban areas respectively. ${ }^{3}$ Almost one third of NAFLD patients develop hyperferritinemia and hepatic iron overload that results in oxidative stress leading to hepatocyte injury, inflammation and fibrosis leading to progression of NAFLD. Also, high body iron levels negatively affect insulin sensitivity by modulating genetic expression thereby increasing insulin resistance. Iron removal improves insulin sensitivity thereby delaying the onset of type 2 diabetes mellitus, improves liver function and NAFLD histology. ${ }^{4}$ Serum ferritin correlates with hepatic iron deposition in patients with NAFLD. Thus, estimation of iron status helps to identify NAFLD patients with increased risk of progression to fibrosis and cirrhosis. Application of iron removal strategies like phlebotomy in such patients might improve IR and NAFLD. There were only a few Indian studies assessing iron metabolism in NAFLD and fewer studies assessing the association of iron status with fibrosis and MetS in NAFLD.

\section{Material and Method}

The study was conducted in the Department of Medicine, GTB Hospital, Delhi from November 2017 - October 2019. It was an analytical, comparative study. Sample size for the study group (cases) and control group were 40 each. Detailed history and clinical examination were done to rule out significant alcohol intake, autoimmune hepatitis and other diseases like Wilson's disease and haemochromatosis. Relevant investigations were done to rule out viral hepatitis. After proper screening, 40 patients in the age group 30-70 years with NAFLD diagnosed on the basis of ultrasound abdomen were selected in the study group. Subjects who are healthy and having no fatty liver on ultrasound were screened and selected in the control group. Ethical clearance was obtained from Institutional Ethics Committee. Written informed consent was taken from all the participants.

Data were obtained from all participants by detailed history and clinical examination, anthropometry, lipid profile, fasting blood sugar levels. Estimation of various parameters of iron status namely serum ferritin, Total Iron Binding Capacity ( $T I B C)$, transferrin saturation, serum iron and serum hepcidin levels were done. $6 \mathrm{ml}$ blood sample was collected after overnight fasting in iron free tube for assessing iron status. Serum ferritin and serum hepcidin were estimated by ELISA. Serum Iron was estimated using the formula:

Serum iron $=223.4 /(O D$ of standard $) \times O D$ of Test, where $O D$ is the optical density.

Total Iron Binding Capacity $(T I B C)=U I B C+$ Serum Iron, where UIBC is the unbound iron binding capacity. Transferrin Saturation was estimated using the formula:

Transferrin saturation (\%) = serum iron/ TIBCX 100.

Iron status of both the groups were compared. The presence of MetS was assessed in NAFLD patients based on International Diabetes Federation (IDF) definition of metabolic syndrome (Box 1). Fibrosis in NAFLD patients was determined using non-invasive scores namely APRI score, FIB-4 and NAFLD fibrosis score. APRI = AST /upper limit of normal AST/ platelet count in $10^{9} / \mathrm{I} \times 100$. An APRI threshold of 1.0 had a sensitivity of $50 \%$ and a specificity of $84 \%$ for advanced fibrosis. FIB 4 Score, Age in years $x$ AST/ (Platelet count in $10^{9} / \mathrm{Lx} \mathrm{ALT}^{1 / 2}$ ). FIB 4 score of $<1.3$ has a low risk of fibrosis, score of 1.3-3.25 has an intermediate risk and a score of $>3.25$ has high risk of fibrosis. NFS = $-1.675+0.037 \times \mathrm{AGE}+0.094 \times \mathrm{BMI}+1.13 \times$ (IFG or diabetes if yes $=1, \mathrm{no}=0)+0.99 \times$ AST/ALT ratio- $0.013 \times$ platelet count -0.66 x albumin, where IFG is impaired fasting glucose. NFS $<-1.455$ has a high negative predictive value for fibrosis. Evaluation for association of iron status with the presence of metabolic syndrome and fibrosis in NAFLD patients was done. Evaluation for association of iron status with the presence of metabolic syndrome and fibrosis in NAFLD patients was done.

\section{Box I: IDF Definition of Metabolic Syndrome}

International Diabetes Federation (IDF) criteria for metabolic syndrome $(2005)^{5}$

Central obesity (defined as waist circumference but can be assumed if $\mathrm{BMI}>30 \mathrm{~kg} / \mathrm{m}^{2}$ ) with ethnicityspecific values, ${ }^{*}$ plus two of the following:

- Triglycerides $150 \mathrm{mg} / \mathrm{dl}$ or greater

- HDL-cholesterol $<40 \mathrm{mg} / \mathrm{dl}$ in men and $<50 \mathrm{mg} /$ $\mathrm{dl}$ in women

- $\quad$ BP $130 / 85 \mathrm{mmHg}$ or greater

- Fasting glucose $100 \mathrm{mg} / \mathrm{dl}$ or greater

*To meet the criteria, waist circumference in Asians must be $>90 \mathrm{~cm}$ in men and $>80 \mathrm{~cm}$ in women.

\section{Statistical Analysis}

Data were entered into Microsoft Excel and analysed using SPSS software $v 20.0$. The level of iron parameters recorded in NAFLD patients and the control group were compared using independent $t$ test and $p$ value of $<0.05$ was considered statistically significant. Assessment of association of various parameters of iron status with the presence of MetS and 
fibrosis in NAFLD patients was done using the Chi-Square test and $p$ valve of $<0.05$ was considered significant.

\section{Result}

In this study, we observed that serum iron level (microgram/ dl) in NAFLD patients expressed as mean \pm SD was 123.88 \pm 24.432 and that in control group it was $107.98 \pm 28.485$. The mean serum iron level in both the groups fell within the normal range but there was a significant difference in the serum iron level in the NAFLD patients and controls ( $p$ value 0.009$)$. The mean serum ferritin level $(\mathrm{ng} / \mathrm{dl}$ ) in the NAFLD cases was $284 \pm 131.122$ and that in the control group was $175.65 \pm 83.038$ and there was significant difference between the serum ferritin level in the NAFLD patients and controls as the $p$ value was $<0.001$ (Figure 1 ). The percentage saturation of transferrin in NAFLD patients (cases) was $46.161 \pm 1.31$ and that in the controls was 38.82 \pm 10 .86. Thus, we observed that mean transferrin saturation in both the groups fell in the normal range, but there was a significant difference in the tranferrin saturation in cases and controls ( $p$ value 0.004 ). We observed that the serum hepcidin $(\mathrm{ng} / \mathrm{ml}$ ) in the NAFLD cases was $42.22 \pm 10.6950$ and that in the control group was $19.595 \pm 13.515$ and there was a significant difference in the serum hepcidin levels in both the groups as the $p$ value was $<0.001$. No significant difference was observed in the TIBC level of NAFLD patients and the controls ( $p$ value - 0.082).

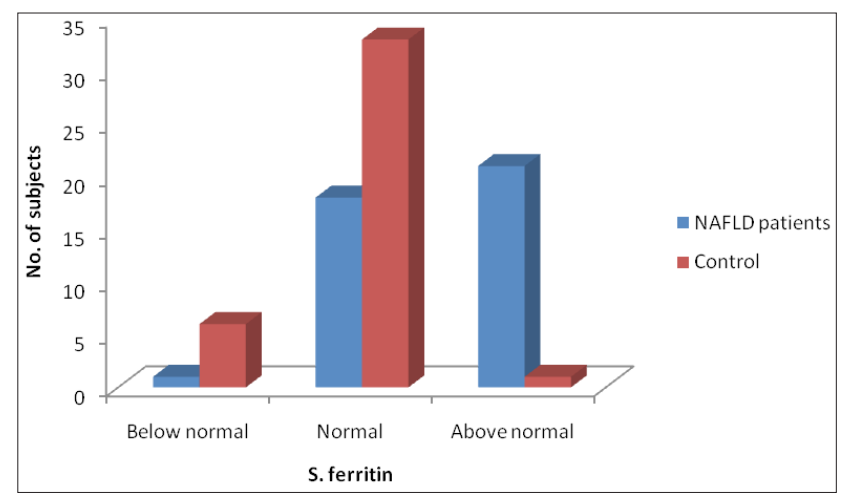

Figure I.Graph Comparing Serum Ferritin in NAFLD Cases and Control Subjects

In this study, $50 \%$ of the NAFLD patients had MetS. The mean values of various parameters of iron status in the patients with MetS and in those without MetS is shown in Table 1.

We observed that there was a significant association of serum ferritin ( $p$ value 0.002 ) with the presence of metabolic syndrome in NAFLD. The other parameters of iron status were not found to have a statistically significant association with MetS in NAFLD.

In NAFLD patients with serum ferritin levels above the normal range, $95.2 \%$ had an APRI of $>1,57.1 \%$ had a FIB
4 score $>3.25,38.1 \%$ had a FIB 4 score in the range 1.30 3.25 and $95.2 \%$ had a NAFLD fibrosis score of $>-1.455$. In NAFLD patients with serum hepcidin above the normal range, $88.9 \%$ had an APRI of $>1,66.7 \%$ had a FIB 4 score of $>3.25,22.2 \%$ had a FIB 4 score of 1.30 to 3.25 and $88.9 \%$ had a NAFLD fibrosis score of $>-1.455$. Thus, in the study, we found that serum ferritin and serum hepcidin in NAFLD patients had a significant association ( $p$ value $<0.05$ ) with fibrosis (APRI, FIB 4 Score and NAFLD fibrosis score). The other parameters of iron status were not found to have significant association with fibrosis.

Table I.Showing the Status of MetS and Mean Values of Parameters of Iron Status

\begin{tabular}{|c|c|c|c|c|}
\hline & $\begin{array}{l}\text { Metabolic } \\
\text { syndrome }\end{array}$ & $\mathbf{N}$ & Mean $\pm S D$ & $\begin{array}{c}\mathbf{p} \\
\text { value }\end{array}$ \\
\hline \multirow{2}{*}{ S.Iron } & Absent & 20 & $115.50 \pm 20.17$ & \multirow[t]{2}{*}{.212} \\
\hline & Present & 20 & $132.25 \pm 25.89$ & \\
\hline \multirow{2}{*}{ S.Ferritin } & Absent & 20 & $\begin{array}{c}219.70 \pm \\
123.09 \\
\end{array}$ & \multirow[t]{2}{*}{.002} \\
\hline & Present & 20 & $349.55 \pm 106.3$ & \\
\hline \multirow{2}{*}{ TIBC } & Absent & 20 & $270.15 \pm 17.48$ & \multirow{2}{*}{.077} \\
\hline & Present & 20 & $272.00 \pm 29.95$ & \\
\hline \multirow{2}{*}{$\begin{array}{l}\text { Transferrin } \\
\text { saturation }\end{array}$} & Absent & 20 & $42.81 \pm 7.25$ & \multirow{2}{*}{.077} \\
\hline & Present & 20 & $49.52 \pm 13.65$ & \\
\hline \multirow{2}{*}{ S.Hepcidin } & Absent & 20 & $40.45 \pm 9.38$ & \multirow{2}{*}{.256} \\
\hline & Present & 20 & $44.00 \pm 11.83$ & \\
\hline
\end{tabular}

\section{Discussion}

Consistent with our study findings, Kowdley et al. demonstrated that hyperferritinemia is common in patients with NAFLD. They observed that a serum ferritin concentration greater than 1.5 times the upper limit of normal was independently associated with advanced fibrosis in NAFLD. ${ }^{6}$

Angulo et al., in their study concluded that serum ferritin levels alone had a low level of diagnostic accuracy for assessing the severity of liver fibrosis in patients with NAFLD.7 A study by Chitturi et al., on 93 patients with $\mathrm{NASH}$, found that serum ferritin concentration was not an independent predictor of advanced fibrosis. ${ }^{8}$ Valenti et al ${ }^{9}$ showed in an Italian cohort of 587 patients with NAFLD that serum ferritin did not predict fibrosis in higher stages (stage $>1$ ).

In a study by Hoki et al., ${ }^{10}$ high levels of hepcidin in NASH were associated with iron-overload due to increased divalent metal transporter 1 expression in enterocytes through increased activity of iron-regulatory protein 1.

Consistent with our findings, several studies showed high ferritin levels and increased prevalence of hyperferritinemia in patients with the whole metabolic syndrome or its individual components, with serum ferritin showing a 
linear increase with the increasing number of features of metabolic syndrome. ${ }^{11-14}$ Zelber-Sagi et al., showed that NAFLD was responsible for the association between serum ferritin and the metabolic syndrome and most of its components suggesting that the relation between serum ferritin and most of metabolic syndrome features might be mediated by the presence of NAFLD at population level. They also suggested that the association found between serum ferritin and components of the metabolic syndrome in the previous studies may be because of the presence of undiagnosed NAFLD in those patients.

Altered regulation of molecules involved in cellular iron export, such as ceruloplasmin and ferroportin 1 induced by inflammation and micronutrients imbalance remains the main mechanism of iron accumulation in NAFLD. Downregulation of the cellular iron exporter fpn-1 is seen in NASH but hepcidin was physiologically increased confirming that altered iron trafficking underlies iron accumulation in NAFLD, whereas preserved hepcidin regulation inhibits fpn1 protein activity, thus, limiting further iron absorption and transferrin saturation. ${ }^{15}$ Increased serum ferritin may also be due to subclinical inflammation and hepatocellular necrosis causing release of ferritin from leukocytes. Increased ferritin levels correlate with IR and increased levels of cytokines. ${ }^{16}$ Another mechanism is that activated Kupffer cells may accumulate iron and release ferritin because of increased erythrophagocytosis, which would cause cytokines release and fibrogenesis. ${ }^{17}$

There are few limitations of this study. The NAFLD patients were selected on the basis of USG evidence and not on the basis of liver biopsy as for major epidemiological studies. USG is widely accepted due to ease of the study and ethical clearance as it is noninvasive. Another limitation of the study was small sample size.

\section{Conclusion}

To conclude, the levels of various parameters of iron status namely serum iron, serum ferritin, transferrin saturation and serum hepcidin were found to be higher in NAFLD patients when compared to controls. There was a significant positive association of serum ferritin with the presence of MetS in NAFLD patients. Also, the study concluded that serum hepcidin and serum ferritin have a significant positive association with fibrosis in NAFLD.

Assessment of iron status in NAFLD may help in early identification of individuals at high risk of fibrosis and other complications like MetS. This would help in timely referral of the patient to tertiary care hospitals for further evaluation like elastography and biopsies. This would also help in early diagnosis and treatment to prevent or to delay the onset of complications like cirrhosis, HCC and death

\section{Conflicts of Interest: None}

\section{References}

1. Chandok N, Minuk G, Wengiel M, Uhanova J. Serum ferritin levels do not predict the stage of underlying non-alcoholic fatty liver disease. J Gastrointestin Liver Dis. 2012 Mar 1;21(1):53-8. [PubMed] [Google Scholar]

2. Dai W, Ye L, Liu A, Wen SW, Deng J, Wu X, Lai Z. Prevalence of nonalcoholic fatty liver disease in patients with type 2 diabetes mellitus: a meta-analysis. Medicine. 2017 Sep;96(39):e8179. [PubMed] [Google Scholar]

3. Das K, Das K, Mukherjee PS, Ghosh A, Ghosh S, Mridha AR, Dhibar T, Bhattacharya B, Bhattacharya D, Manna B, Dhali GK. Nonobese population in a developing country has a high prevalence of nonalcoholic fatty liver and significant liver disease. Hepatology. 2010 May;51(5):1593-602. [Google Scholar]

4. Zelber-Sagi S, Nitzan-Kaluski D, Halpern Z, Oren R. NAFLD and hyperinsulinemia are major determinants of serum ferritin levels. Jhepatol. 2007 Apr 1;46(4):700-7. [PubMed] [Google Scholar]

5. Alberti KG, Zimmet P, Shaw J: The metabolic syndrome-a new worldwide definition. Lancet 2005, 366:1059-1062. [PubMed] [Google Scholar]

6. Kowdley KV, Belt P, Wilson LA, Yeh MM, NeuschwanderTetri BA, Chalasani N, Sanyal AJ, Nelson JE, NASH Clinical Research Network. Serum ferritin is an independent predictor of histologic severity and advanced fibrosis in patients with nonalcoholic fatty liver disease. Hepatology. 2012 Jan;55(1):77-85. [PubMed] [Google Scholar]

7. Angulo P, George J, Day CP, Vanni E, Russell L, Anna C, Liaquat H, Mezzabotta L, Lee E, Bugianesi E. Serum ferritin levels lack diagnostic accuracy for liver fibrosis in patients with nonalcoholic fatty liver disease. Clin Gastroenterol Hepatol. 2014 Jul 1;12(7):1163-9. [PubMed] [Google Scholar]

8. Chitturi S, Weltman M, Farrell GC, McDonald D, Liddle C, Samarasinghe D, Lin R, Abeygunasekera S, George J. HFE mutations, hepatic iron, and fibrosis: ethnic-specific association of NASH with $\mathrm{C} 282 \mathrm{Y}$ but not with fibrotic severity. Hepatology. 2002 Jul;36(1):142-9. [PubMed] [Google Scholar]

9. Valenti L, Fracanzani AL, Bugianesi E, Dongiovanni $P$, Galmozzi E, Vanni E, Canavesi E, Lattuada E, Roviaro G, Marchesini G, Fargion S. HFE genotype, parenchymal iron accumulation, and liver fibrosis in patients with nonalcoholic fatty liver disease. Gastroenterology. 2010 Mar 1;138(3):905-12. [PubMed] [Google Scholar]

10. Hoki T, Miyanishi K, Tanaka S, Takada K, Kawano Y, Sakurada A, Sato M, Kubo T, Sato T, Sato Y, Takimoto R. Increased duodenal iron absorption through up-regulation of divalent metal transporter 1 from enhancement of iron regulatory protein 1 activity in patients with nonalcoholic steatohepatitis. Hepatology. 
2015 Sep;62(3):751-61. [PubMed] [Google Scholar]

11. Bozzini C, Girelli D, Olivieri O, Martinelli N, Bassi A, De Matteis G, Tenuti I, Lotto V, Friso S, Pizzolo F, Corrocher R. Prevalence of body iron excess in the metabolic syndrome. Diabetes Care. 2005 Aug 1;28(8):2061-3. [PubMed] [Google Scholar]

12. Jehn M, Clark JM, Guallar E. Serum ferritin and risk of the metabolic syndrome in US adults. Diabetes Care. 2004 Oct 1;27(10):2422-8. [PubMed] [Google Scholar]

13. Tuomainen TP, Nyyssönen $K$, Salonen $R$, Tervahauta A, Korpela H, Lakka T, Kaplan GA, Salonen JT. Body iron stores are associated with serum insulin and blood glucose concentrations: population study in 1,013 eastern Finnish men. Diabetes Care. 1997 Mar 1;20(3):426-8. [PubMed] [Google Scholar]

14. Piperno A, Trombini P, Gelosa M, Mauri V, Pecci V, Vergani A, Salvioni A, Mariani R, Mancia G. Increased serum ferritin is common in men with essential hypertension. J Hypertens. 2002 Aug 1;20(8):1513-8. [PubMed] [Google Scholar]

15. Aigner E, Theurl I, Theurl M, Lederer D, Haufe H, Dietze O, Strasser M, Datz C, Weiss G. Pathways underlying iron accumulation in human nonalcoholic fatty liver disease. The Am J Clin Nutr. 2008 May 1;87(5):137483. [PubMed] [Google Scholar]

16. Manco M, Alisi A, Real JM, Equitani F, DeVito R, Valenti L, Nobili V. Early interplay of intra-hepatic iron and insulin resistance in children with non-alcoholic fatty liver disease. J Hepatol. 2011 Sep 1;55(3):647-53. [PubMed] [Google Scholar]

17. Otogawa K, Kinoshita K, Fujii H, Sakabe M, Shiga R, Nakatani K, Ikeda K, Nakajima Y, Ikura Y, Ueda M, Arakawa T. Erythrophagocytosis by liver macrophages (Kupffer cells) promotes oxidative stress, inflammation, and fibrosis in a rabbit model of steatohepatitis: implications for the pathogenesis of human nonalcoholic steatohepatitis. Am J Pathol. 2007 Mar 1;170(3):96780. [PubMed] [Google Scholar] 